\title{
Angiosperms, Pachaiyappa's College, Chennai, Tamil Nadu, India
}

\author{
Muthulingam Udayakumar, Muniappan Ayyanar and Thangavel Sekar*
}

\author{
Pachaiyappa's College, Post Graduate and Research Department of Botany, Division of Biodiversity and Biotechnology. Chennai, 600 030, TN, India. \\ * Corresponding author. E-mail: tsekar_bot@yahoo.com
}

\begin{abstract}
We provide a checklist of Angiosperms along with the details of life form from a $~ 9.6$ ha of non-concreted area of Pachaiyappa's College (PC) campus, Chennai, Tamil Nadu state, India. This area harbors 256 species belonging to 212 genera in 71 families. Families with maximum number of species include Fabaceae (31 species) followed by Malvaceae (15), Euphorbiaceae (13), Apocynaceae (12), Acanthaceae and Poaceae (11 each), Bignoniaceae and Rubiaceae (eight each) and Arecaceae, Moraceae, Rutaceae and Verbenaceae (seven each). The surveyed area represents a remnant of tropical dry evergreen forest (TDEF), as a substantial number of species collected in the present study belong exclusively to the Coromandel Coast (CC) TDEFs. PC is still preserving the biodiversity by means of strict rules and regulations enforced for the maintenance of the college premises.
\end{abstract}

\section{INTRODUCTION}

The forest types of Coromandel Coast (CC) of peninsular India include tropical dry evergreen forests (TDEFs), dry evergreen scrubs and mangroves (Champion and Seth 1968). The Coromandel coastal plains extend about 80-100 $\mathrm{km}$ inland from the coast (Mani 1974). Floristically, TDEF is distinguished by a fair representation of characteristic and preferential species, exclusively or mostly confined to this vegetation type (Champion and Seth 1968; MeherHomji 1974). The tropical dry evergreen forests occurring in patches, short-statured, largely three-layered, tree dominated evergreen forests with a sparse and patchy ground flora (Venkateswaran and Parthasarathy 2003). Invariably the TDEF patches are protected by the local people as sacred groves (SGs) (Parthasarathy et al. 2008; Udayakumar and Parthasarathy 2010). TDEFs have been distinguished from other forest types by various authors (Sebastine and Ellis 1967; Champion and Seth 1968; Rao and Meher-Homji 1993) and they represent a peculiar type, confined to the southeastern coast of India, northwest Sri Lanka (Blasco and Legris 1973), northeastern Thailand (Bunyavejechewin 1999) and Jamaica (Kelly et al. 1988). As to the inventory of plant biodiversity of TDEFs of CC, Parthasarathy and Karthikeyan (1997) listed a total of 54 woody plant species in 47 genera and 31 families from two sacred groves of Cuddalore district; Reddy and Parthasarathy (2003) documented 39 (34 genera and 24 families) woody liana species from four sacred groves; Venkateswaran and Parthasarathy (2003) documented the presence of 46 woody flowering plant species (43 genera in 25 families) from two sacred groves; Mani and Parthasarathy (2005) recognized 60 tree species (49 genera in 24 families) from five sacred groves in Pudukottai district of Tamil Nadu; Venkateswaran and Parthasarathy (2005) identified 29 tree species (26 genera in 26 families) from a sacred grove of Villupuram district of Tamil Nadu.

Urban green space that includes streets with trees, parks, vegetated colleges and schools plays vital role in conservation of local environment. It can decrease the urban island heat effect (Chow and Roth 2006), storm water run-off and flooding (Mc Pherson et al. 1997). Trees of the concretized urban environment render food to birds and other city dwelling animals (Fernandez-Zuricic 2000). They act as noise filters, air purifiers, sequester carbon and pollutant traps (Mc Pherson 1997; Beckett et al. 2000). Urban greening and urban forests are particularly important to healthy cities in developing countries (Thaiutsa et al. 2008). Due to escalating urbanization, green space and urban trees become increasingly important in developing countries (Cy 2006). Documentation of existing green spaces of the urban environment is important to determine existing resources and to set target for future improvements (Miller 1996). Results of tree inventory and assessment of urban environment can be a useful tool in urban planning and conservation of important tree species (Cy 2006). Urbanization is one of the major reasons for destruction of the natural vegetation. Urbanized areas can also harbor a high number of threatened species (Sodhi et al. 2010). Considering the importance of enumeration of plants, particularly in a typical metropolitan area such as Chennai, we made a qualitative floristic survey and prepared a checklist of angiosperm species of Pachaiyappa's College, Chennai which has a protected patch of the Coromandel Coast TDEFs.

\section{MATERIALS AND METHODS}

Study site

Qualitative angiosperm floristic survey was carried out in about 9.6 ha of Pachaiyappa's College $\left(13^{\circ} 07^{\prime} 30^{\prime \prime}\right.$ $\mathrm{N}, 80^{\circ} 23^{\prime} 31^{\prime \prime} \mathrm{E}$ ), established in the year 1842 , and one of the oldest and famous institutions of Higher learning in Tamil Nadu. It is located in middle of the Chennai, the most populated, metropolitan and capital city of Tamil Nadu state, south India. The total area of the College is about $\sim 16$ ha and it is a part of the Coromandel Coast. The city is experiencing tropical dissymmetric climate and receiving 
bulk of rainfall during the north-east monsoon (OctoberDecember). Average annual rainfall received by the city is $\sim 1,300 \mathrm{~mm}$. The average temperature is $37^{\circ} \mathrm{C}$ in summer and $24{ }^{\circ} \mathrm{C}$ in winter (Chennai District 2009). The east of the city is lined up by the sea shore of Bay of Bengal and the north, west and south are bound by land (Thiruvallur district) (Figure 1).

\section{Data collection}

During March-June 2009 enumeration of angiosperm flora was carried out by qualitative floristic survey in about 60 days in a total of $\sim 9.6$ ha. Species were identified using regional floras (Gamble 1921-1935; Matthew 1991; Nair et al. 1983; Henry et al. 1987; 1989). Angiosperm Phylogeny Group II was followed to classify the species. For all documented species the binomial and author citation were checked thoroughly with IPNI (International Plant Names Index). The well preserved specimens with voucher number were deposited in Herbarium of Post Graduate and Research Department of Botany, Pachaiyappa's College, Chennai, Tamil Nadu, India.

\section{RESULTS AND Discussion}

This qualitative floristic survey revealed the presence of 256 Angiosperm species belonging to 212 genera in 71 families. Plant binomial, family, life form and voucher number are provided in Table 1 . The most speciose families include Fabaceae (31 species) followed by Malvaceae (15), Euphorbiaceae (13), Apocynaceae (12), Acanthaceae and Poaceae (11 each), Bignoniaceae and Rubiaceae (8 each) and Arecaceae, Moraceae, Rutaceae and Verbenaceae (7 each), whereas 29 families represented by a single species which include Aristolochiaceae, Bixaceae, Caricaceae, Celastraceae, Cornaceae and Clusiaceae etc., (Table 1). Among life-forms trees dominate the campus with 99 species followed by herbs (76), shrubs (51), lianas (22) and herbaceous climbers (8). Dicotyledons are more common with $222(66.72 \%)$ species followed by monocotyledons with 34 (13.28\%) species. Mayuranathan (1994) reported 1039 species (843 dicotyledons and 196 monocotyledons) of flowering plants from the entire Chennai district. Giles Lal and Livingstone (1978) have documented 458 (256 woody plants and 202 herbs) flowering plant species from ca. 151 ha campus of an age-old Madras Christian College (MCC), Chennai. Fabaceae is the dominant family in Chennai district, MCC as well as PC with 87, 59 and 31 species respectively. Presence of Cordia obliqua, Ecbolium viride, Ixora pavetta, Pavetta indica, Pterospermum canescens, Sansevieria roxburghiana and Streblus asper confirms that the flora of the area studied is a remnant of tropical dry evergreen forests of Coromandel Coast.

Among the plant species identified from PC, Delonix regia (IUCN 2009; Status: Vulnerable B1+2c ver. 2.3), Guaiacum officinale (IUCN 2009; Status: Endangered C2a ver. 2.3), Pterospermum canescens and Sansevieria roxburghiana (endemic to $\mathrm{CC}$, Parthasarathy et al. 2008), are the most important species from the point of conservation. Important plant species are featured in Figures 2-6. Eight decades ago Cordia obliqua, Evolvulus nummularius and Spathodea companulata were only present at PC (Mayuranathan 1929), but now these species are distributed throughout the city. Cordia obliqua

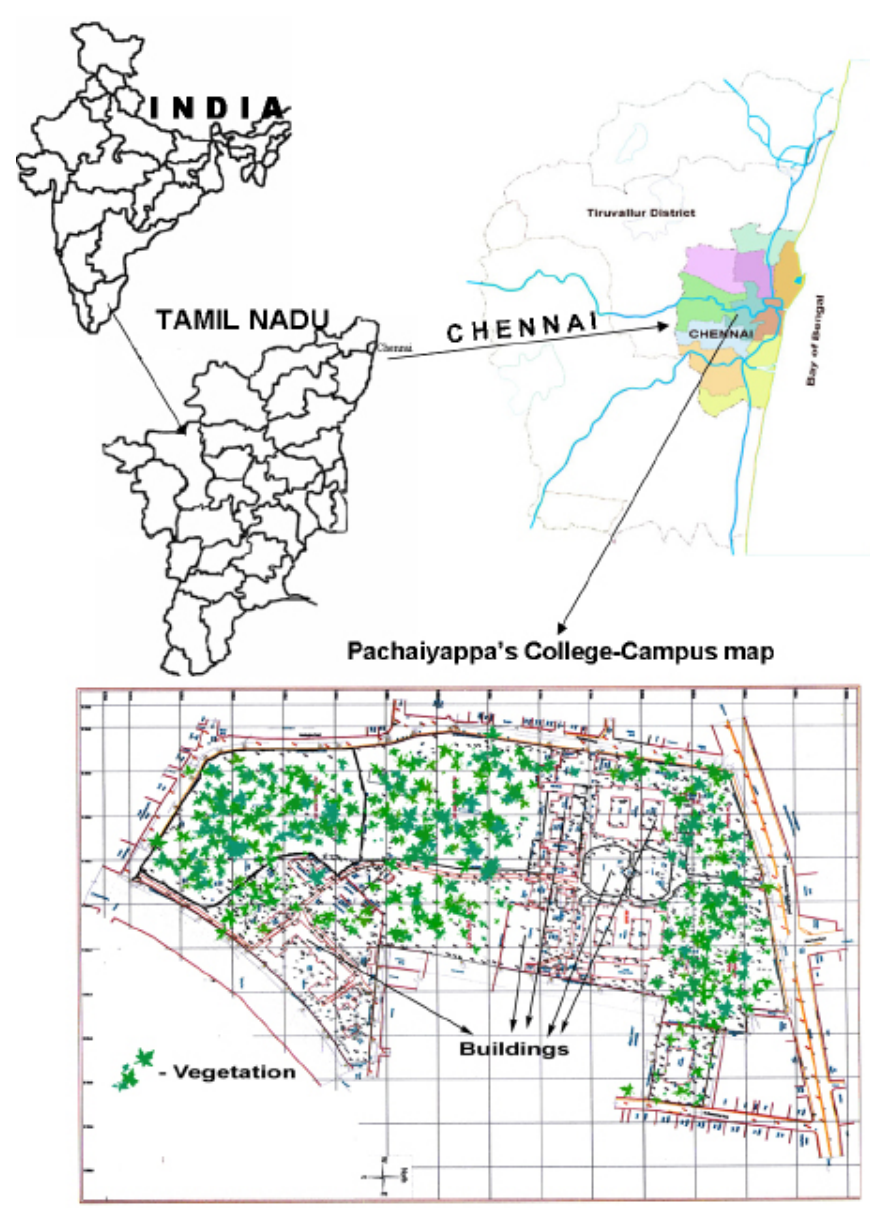

FigurE 1. Map showing the vegetation cover of the Pachaiyappa's College campus, Chennai, Tamil Nadu, India.

and Spathodea companulata are extensively planted as avenue tree, and Evolvulus nummularius spreads as a weed. Previously, Fyson (1921) reported 100 flowering plant species from whole Chennai district of which 55 species are available in the PC. The earlier works on TDEFs of CC has revealed the presence of 149 (122 genera and 49 families) species of Angiosperms including 42 woody liana and 102 tree species (Parthasarathy et al. 2008); Recently, Udayakumar and Parthasarathy (2010) recorded 312 (252 genera and 80 families) species of flowering plants from 86 sacred groves of southern Coromandel Coast of India.

As reported by Mani (1974) that the CC extended up to 50-100 km inland from the coast, the study area is located $5 \mathrm{~km}$ inland from the Coast of Chennai constituting a part of the CC TDEFs. In Chennai itself such CC TDEFs patches are well protected in the Guindy National park and the Raj Bhavan, Chennai (Tamil Nadu Government 2009). As of now, approximately $40 \%$ (6.4 ha) of the College land is occupied by buildings and play grounds and the remaining $60 \%$ (9.6 ha) supports the life of various herbs, herbaceous climbers, shrubs, woody lianas and trees. Though the PC is located in the Chennai Metropolis, the biodiversity of the campus is protected by strict rules and regulations. Many of the introduced and exotic-ornamental plants are also growing along with the native flora to enhance the aesthetic value and biodiversity wealth of the campus. Documentation of plant biodiversity of the colleges is also an essential factor that promotes to evaluate the total biodiversity wealth of any particular place such as town, city, district etc. 



Figure 2. A) Undisturbed tree stand of Pachaiyappa's College campus; B) Allophylus cobbe (Sapindaceae); C) Bougainvillea spectabilis (Nyctaginaceae); D) Coccoloba uvifera (Polygonaceae); E) Cordia obliqua (Boraginaceae); F) Cordia sebestena (Boraginaceae). 

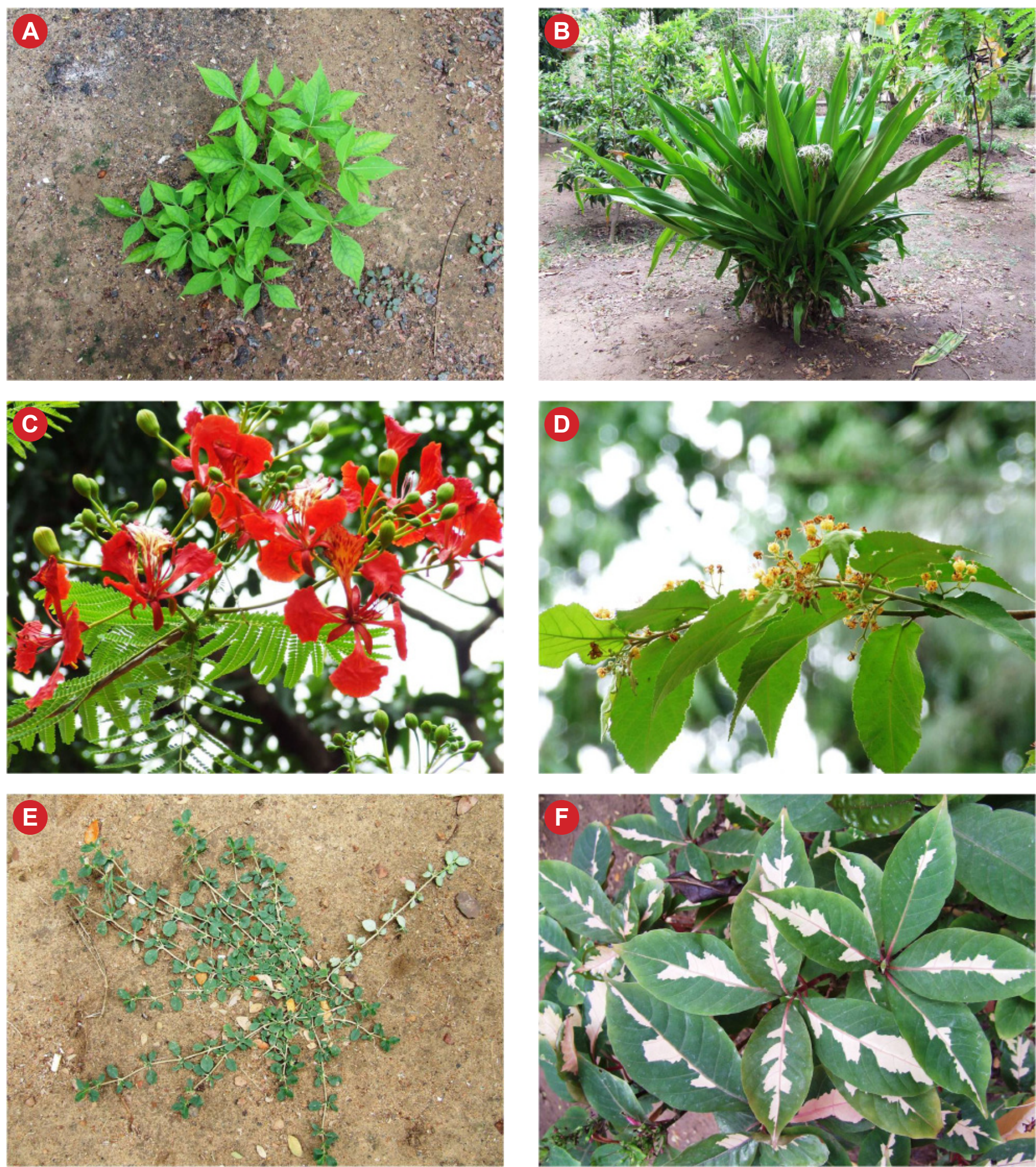

Figure 3. A) Crateva magna (Brassicaceae); B) Crinum asiaticum (Amaryllidaceae); C) Delonix regia (Fabaceae); D) Guazuma ulmifolia (Malvaceae); E) Gisekia pharnaceoides (Gisekiaceae); F) Graptophyllum hortense (Acanthaceae). 

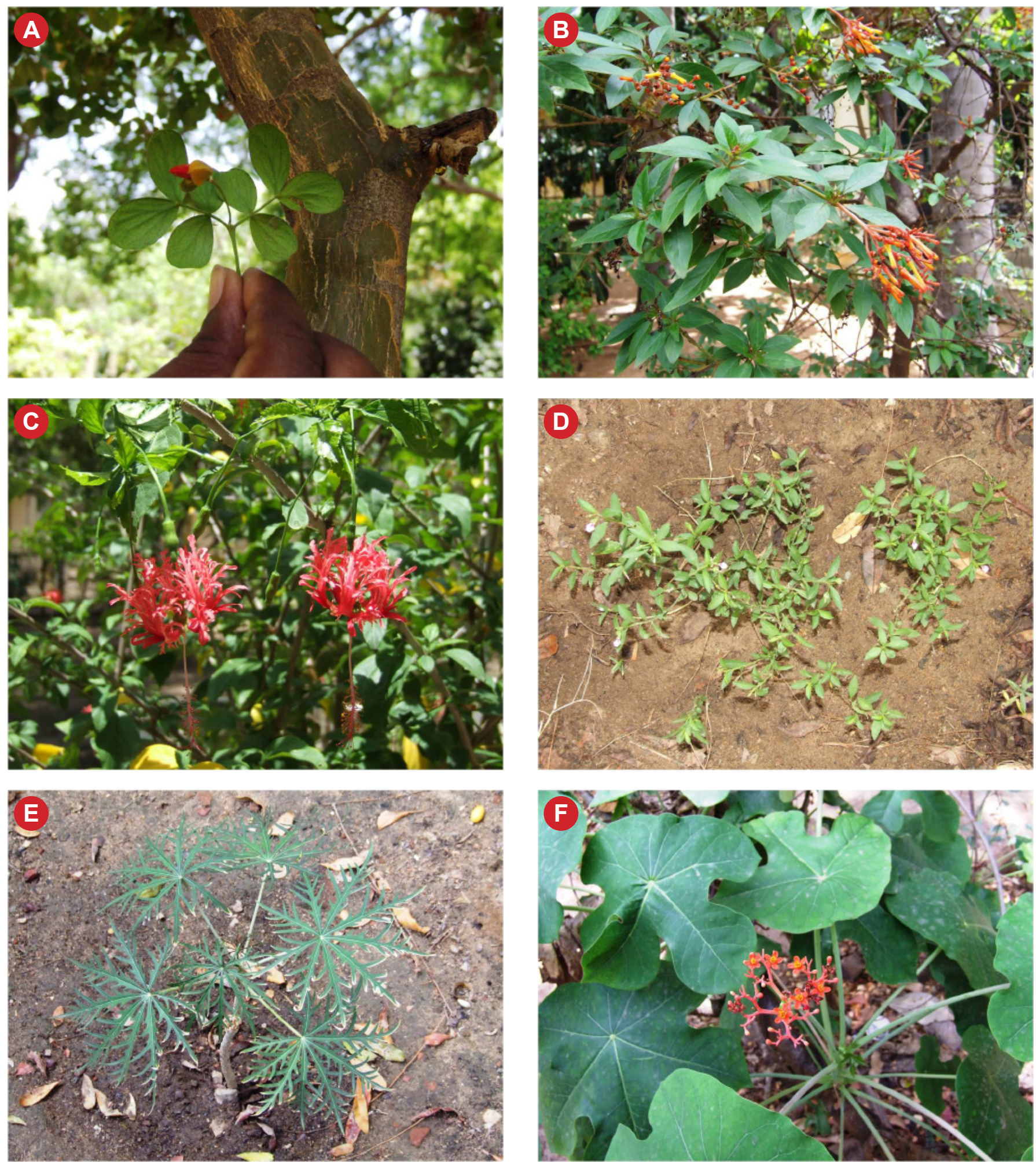

Figure 4. A) Guaiacum officinale (Zygophyllaceae); B) Hamelia patens (Rubiaceae); C) Hibiscus schizopetalus (Malvaceae); D) Hybanthus enneaspermus (Violaceae); E) Jatropha multifida (Euphorbiaceae); F) Jatropha podagrica (Euphorbiaceae). 

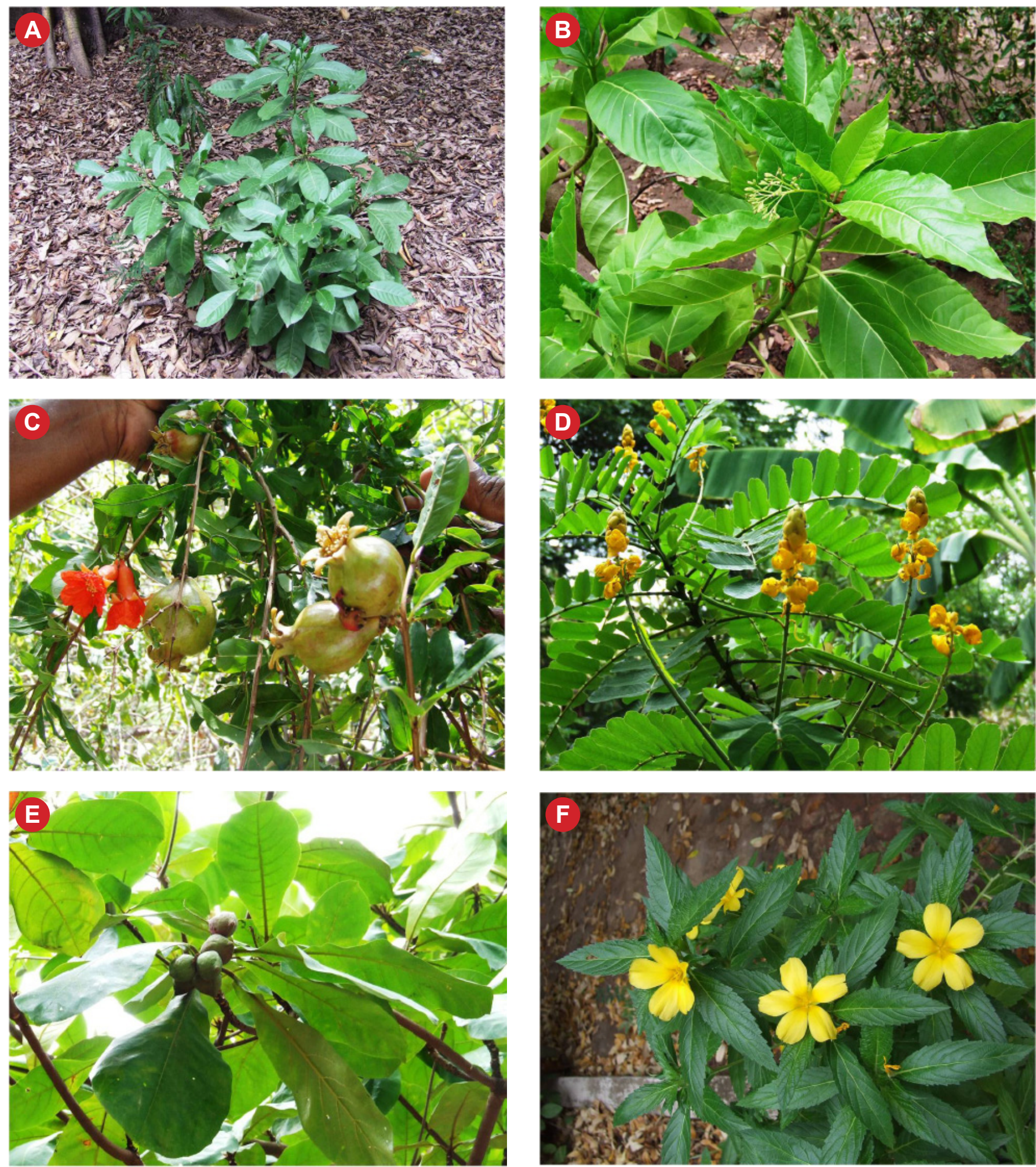

Figure 5. A) Pavetta indica (Rubiaceae); B) Pisonia alba (Nyctaginaceae); C) Punica granatum (Lythraceae); D) Senna alata (Fabaceae); E) Terminalia catappa (Combretaceae); F) Turnera ulmifolia (Turneraceae). 

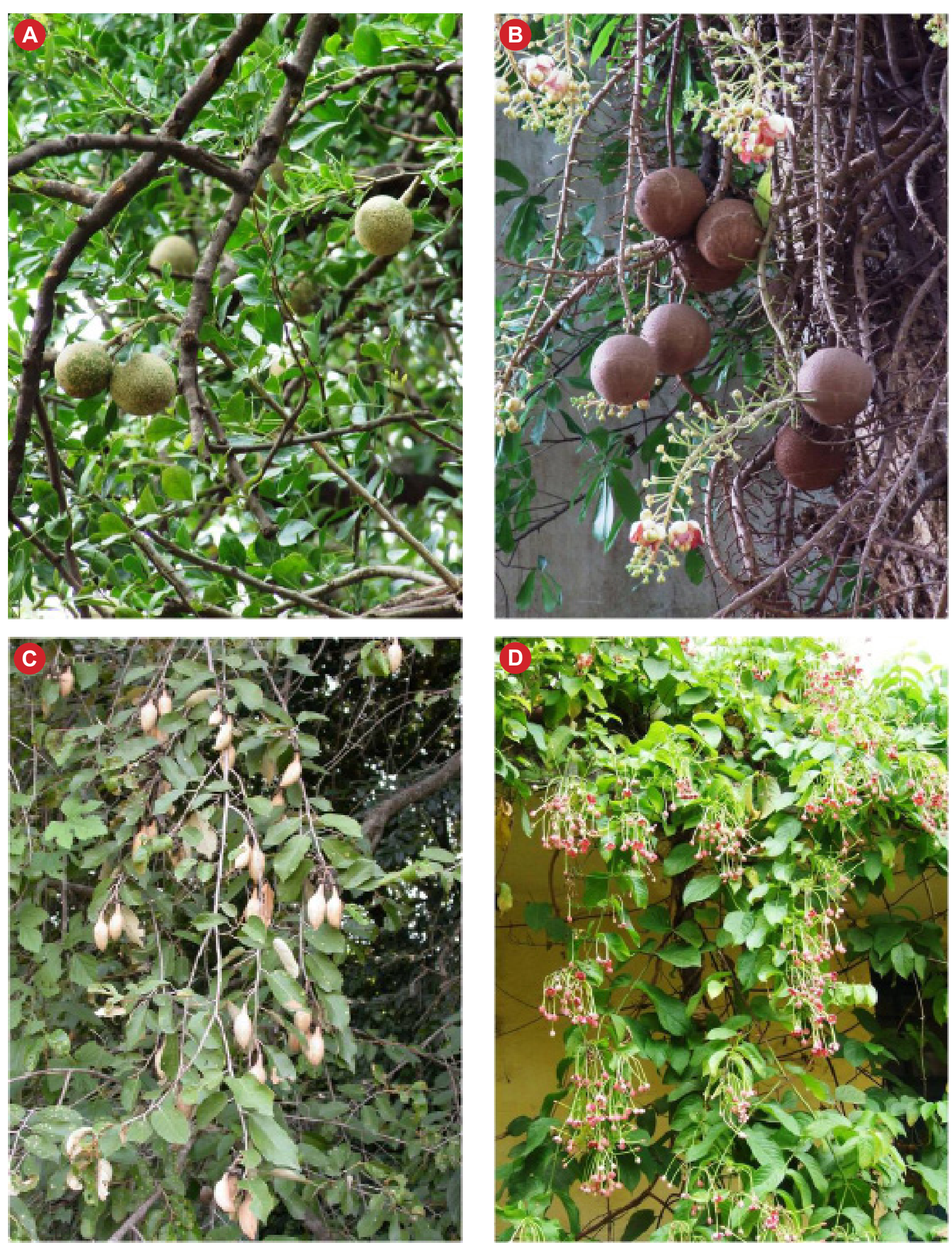

Figure 6. A) Limonia acidissima (Rutaceae); B) Couroupita guianensis (Lecythidaceae); C) Pterospermum canescens (Malvaceae); D) Quisqualis indica (Combretaceae). 
TABLE 1. Angiosperm plant species from Pachaiyappa's College, Chennai, Tamil Nadu, India. The life-form was also presented $(\mathrm{H}=\mathrm{Herb}$; $\mathrm{HC}=$ Herbaceous climber; $\mathrm{L}=$ Liana; $\mathrm{S}=$ Shrub; $\mathrm{T}=$ Tree).

\begin{tabular}{|c|c|c|}
\hline TAXON & $\begin{array}{l}\text { LIFE- } \\
\text { FORM }\end{array}$ & $\begin{array}{c}\text { VOUCHER } \\
\text { NO. }\end{array}$ \\
\hline \multicolumn{3}{|l|}{ ACANTHACEAE } \\
\hline Andrographis echioides Nees & $\mathrm{H}$ & 1755 \\
\hline Barleria cristata $\mathrm{L}$. & $\mathrm{S}$ & 1532 \\
\hline Dipteracanthus prostratus Nees & $\mathrm{H}$ & 1588 \\
\hline Ecbolium viride (Forssk.) Alston & $\mathrm{H}$ & 1591 \\
\hline Graptophyllum hortense Nees & S & 1609 \\
\hline Justicia adhatoda L. & $\mathrm{S}$ & 1631 \\
\hline Justicia gendarussa Burm.f & $\mathrm{S}$ & 1632 \\
\hline Justicia procumbens L. & $\mathrm{H}$ & 1633 \\
\hline Justicia tranquebariensis L.f. & $\mathrm{H}$ & 1634 \\
\hline Ruellia tuberosa L. & $\mathrm{H}$ & 1693 \\
\hline Thunbergia grandiflora Roxb. & $\mathrm{L}$ & 1717 \\
\hline \multicolumn{3}{|l|}{ AIzoACEAE } \\
\hline Trianthema portulacastrum $\mathrm{L}$. & $\mathrm{H}$ & 1723 \\
\hline \multicolumn{3}{|l|}{ AMARANTHACEAE } \\
\hline Achyranthes aspera L. & $\mathrm{H}$ & 1506 \\
\hline Aerva lanata (L.) Juss. Ex Schult. & $\mathrm{H}$ & 1510 \\
\hline Alternanthera paronychioides A.St.-Hil. & $\mathrm{H}$ & 1516 \\
\hline Amaranthus spinosus $\mathrm{L}$. & $\mathrm{H}$ & 1517 \\
\hline Gomphrena procumbens Zuccagni. & $\mathrm{H}$ & 1608 \\
\hline Amaranthus viridis $\mathrm{L}$. & $\mathrm{H}$ & 1518 \\
\hline \multicolumn{3}{|l|}{ AMARYLLIDACEAE } \\
\hline Crinum asiaticum L. & $\mathrm{S}$ & 1579 \\
\hline Curculigo orchioides Gaertn. & $\mathrm{H}$ & 1580 \\
\hline \multicolumn{3}{|l|}{ ANACARDIACEAE } \\
\hline Lannea coromandelica (Houtt.) Merr. & $\mathrm{T}$ & 1637 \\
\hline Mangifera indica $\mathrm{L}$. & $\mathrm{T}$ & 1645 \\
\hline \multicolumn{3}{|l|}{ ANNONACEAE } \\
\hline Annona squamosa L. & $\mathrm{T}$ & 1519 \\
\hline Artabotrys hexapetalus (L.f.) Bhandari & S & 1522 \\
\hline Polyalthia longifolia (Sonn.) Thwaites & $\mathrm{T}$ & 1680 \\
\hline \multicolumn{3}{|l|}{ APOCYNACEAE } \\
\hline Adenium obesum Roem. \& Schult. & $\mathrm{S}$ & 1508 \\
\hline Alstonia scholaris (L.) R.Br. & $\mathrm{T}$ & 1515 \\
\hline Calotropis gigantea (L.) W.T.Aiton & $\mathrm{S}$ & 1546 \\
\hline Catharanthus roseus (L.) G.Don & $\mathrm{H}$ & 1559 \\
\hline Decalepis hamiltonii Wight \& Arn. & $\mathrm{L}$ & 1585 \\
\hline Hemidesmus indicus (L.) R.Br. & $\mathrm{L}$ & 1617 \\
\hline Nerium oleander L. & S & 1662 \\
\hline Pergularia daemia (Forssk.) Chiov. & $\mathrm{L}$ & 1670 \\
\hline Rauvolfia tetraphylla L. & $\mathrm{H}$ & 1687 \\
\hline Secamone emetica (Retz.) Schult. & $\mathrm{L}$ & 1696 \\
\hline Tylophora indica Merr. & $\mathrm{L}$ & 1729 \\
\hline Wrightia tinctoria R.Br. & $\mathrm{T}$ & 1733 \\
\hline \multicolumn{3}{|l|}{ ARACEAE } \\
\hline Colocasia antiquorum Schott & $\mathrm{S}$ & 1572 \\
\hline Epipremnum pinnatum (L.) Engl. & $\mathrm{L}$ & 1592 \\
\hline ARECACEAE & & \\
\hline
\end{tabular}

TABle 1. ContinUed.

\begin{tabular}{|c|c|c|}
\hline TAXON & $\begin{array}{l}\text { LIFE- } \\
\text { FORM }\end{array}$ & $\begin{array}{c}\text { VOUCHER } \\
\text { NO. }\end{array}$ \\
\hline Borassus flabellifer $\mathrm{L}$. & $\mathrm{T}$ & 1539 \\
\hline Caryota urens L. & $\mathrm{T}$ & 1553 \\
\hline Chrysalidocarpus lutescens H.Wendl. & $\mathrm{T}$ & 1760 \\
\hline Cocos nucifera L. & $\mathrm{T}$ & 1570 \\
\hline Livistona chinensis $\mathrm{R} . \mathrm{Br}$. & $\mathrm{T}$ & 1643 \\
\hline Phoenix sylvestris Roxb. & $\mathrm{T}$ & 1671 \\
\hline Roystonea regia (Kunth) O.F.Cook & $\mathrm{T}$ & 1692 \\
\hline \multicolumn{3}{|l|}{ ARISTOLOCHIACEAE } \\
\hline Aristolochia bracteolata Lam. & $\mathrm{H}$ & 1521 \\
\hline \multicolumn{3}{|l|}{ ASPARAGACEAE } \\
\hline Asparagus racemosus Willd. & $\mathrm{L}$ & 1524 \\
\hline Sansevieria roxburghiana Schult.f. & $\mathrm{H}$ & 1694 \\
\hline \multicolumn{3}{|l|}{ ASTERACEAE } \\
\hline Acanthospermum hispidum DC. & $\mathrm{H}$ & 1751 \\
\hline Ageratum conyzoides L. & $\mathrm{H}$ & 1749 \\
\hline Blumea wightiana DC. & $\mathrm{H}$ & 1750 \\
\hline Tridax procumbens L. & $\mathrm{H}$ & 1726 \\
\hline Vernonia cinerea (L.) Less. & $\mathrm{H}$ & 1748 \\
\hline \multicolumn{3}{|l|}{ BIGNONIACEAE } \\
\hline Bignonia capreolata L. & $\mathrm{L}$ & 1534 \\
\hline Crescentia cujeta L & $\mathrm{T}$ & 1762 \\
\hline Kigelia africana (Lam.) Benth. & $\mathrm{T}$ & 1636 \\
\hline Markhamia stipulata Seem. & $\mathrm{T}$ & 1649 \\
\hline Millingtonia hortensis L.f. & $\mathrm{T}$ & 1653 \\
\hline Spathodea campanulata P.Beauv. & $\mathrm{T}$ & 1703 \\
\hline Tabebuia rosea DC. & $\mathrm{T}$ & 1709 \\
\hline Tecoma stans (L.) Kunth & $\mathrm{S}$ & 1711 \\
\hline \multicolumn{3}{|l|}{ BIXACEAE } \\
\hline Bixa orellana $\mathrm{L}$. & $\mathrm{T}$ & 1535 \\
\hline \multicolumn{3}{|l|}{ BORAGINACEAE } \\
\hline Carmona retusa (Vahl) Masam. & $\mathrm{S}$ & 1552 \\
\hline Cordia obliqua Willd. & $\mathrm{T}$ & 1574 \\
\hline Cordia sebestena L. & $\mathrm{T}$ & 1575 \\
\hline Heliotropium indicum $\mathrm{L}$. & $\mathrm{H}$ & 1616 \\
\hline Trichodesma indicum R.Br. & $\mathrm{H}$ & 1725 \\
\hline \multicolumn{3}{|l|}{ BRASSICACEAE } \\
\hline Capparis decidua Edgew. & $\mathrm{S}$ & 1548 \\
\hline Capparis sepiaria L. & $\mathrm{S}$ & 1549 \\
\hline Cleome viscosa $\mathrm{L}$. & $\mathrm{H}$ & 1565 \\
\hline Crateva magna DC. & $\mathrm{T}$ & 1578 \\
\hline Gynandropsis gynandra (L.) Briq. & $\mathrm{H}$ & 1613 \\
\hline \multicolumn{3}{|l|}{ CARICACEAE } \\
\hline Carica papaya L. & $\mathrm{T}$ & 1551 \\
\hline \multicolumn{3}{|l|}{ CASUARINACEAE } \\
\hline Casuarina equisetifolia $\mathrm{L}$. & $\mathrm{T}$ & 1557 \\
\hline Casuarina torulosa Aiton & $\mathrm{T}$ & 1558 \\
\hline \multicolumn{3}{|l|}{ Celastraceae } \\
\hline Celastrus paniculatus Willd. & $\mathrm{L}$ & 1560 \\
\hline
\end{tabular}


TABLE 1. ContinUed.

\begin{tabular}{|c|c|c|}
\hline TAXON & $\begin{array}{l}\text { LIFE- } \\
\text { FORM }\end{array}$ & $\begin{array}{c}\text { VOUCHER } \\
\text { NO. }\end{array}$ \\
\hline Calophyllum inophyllum L. & $\mathrm{T}$ & 1545 \\
\hline \multicolumn{3}{|l|}{ Colchicaceae } \\
\hline Gloriosa superba L. & $\mathrm{HC}$ & 1605 \\
\hline \multicolumn{3}{|l|}{ COMBRETACEAE } \\
\hline Quisqualis indica L. & $\mathrm{L}$ & 1686 \\
\hline Terminalia bellirica (Gaertn.) Roxb. & $\mathrm{T}$ & 1714 \\
\hline Terminalia catappa $\mathrm{L}$. & $\mathrm{T}$ & 1715 \\
\hline \multicolumn{3}{|l|}{ COMMELINACEAE } \\
\hline Commelina benghalensis L. & $\mathrm{T}$ & 1573 \\
\hline Cyanotis axillaris (L.) D.Don & $\mathrm{H}$ & 1756 \\
\hline Tradescantia discolor Raf. & $\mathrm{H}$ & 1721 \\
\hline \multicolumn{3}{|l|}{ CONVOLVULACEAE } \\
\hline Evolvulus nummularius (L.) L. & $\mathrm{H}$ & 1763 \\
\hline Ipomoea aquatica Forssk. & $\mathrm{HC}$ & 1623 \\
\hline Ipomoea sepiaria J.Koenig ex Roxb. & $\mathrm{HC}$ & 1752 \\
\hline \multicolumn{3}{|l|}{ CORNACEAE } \\
\hline Alangium salviifoliium (L.f.) Wangerin & $\mathrm{T}$ & 1511 \\
\hline \multicolumn{3}{|l|}{ Costaceae } \\
\hline Costus speciosus $\mathrm{Sm}$. & $\mathrm{H}$ & 1576 \\
\hline \multicolumn{3}{|l|}{ Crassulaceae } \\
\hline Kalanchoe pinnata (Lam.) Pers. & $\mathrm{H}$ & 1635 \\
\hline \multicolumn{3}{|l|}{ Cucurbitaceae } \\
\hline Coccinia grandis (L.) Voigt & $\mathrm{L}$ & 1568 \\
\hline Mukia maderaspatana (L.) M.Roem. & $\mathrm{HC}$ & 1658 \\
\hline \multicolumn{3}{|l|}{ CYPERACEAE } \\
\hline Cyperus alternifolius L. & $\mathrm{H}$ & 1582 \\
\hline Cyperus rotundus $\mathrm{L}$. & $\mathrm{H}$ & 1583 \\
\hline Kyllinga monocephala L.f. & $\mathrm{H}$ & 1757 \\
\hline \multicolumn{3}{|l|}{ EUPHORBIACEAE } \\
\hline Acalypha amentacea Roxb. & $\mathrm{S}$ & 1504 \\
\hline Acalypha indica L. & $\mathrm{H}$ & 1505 \\
\hline Codiaeum variegatum (L.) A.Juss. & $\mathrm{S}$ & 1571 \\
\hline Euphorbia heterophylla L. & $\mathrm{H}$ & 1594 \\
\hline Euphorbia hirta L. & $\mathrm{H}$ & 1595 \\
\hline Jatropha curcas L. & $\mathrm{S}$ & 1627 \\
\hline Jatropha gossypifolia L. & $\mathrm{S}$ & 1628 \\
\hline Jatropha multifida L. & $\mathrm{S}$ & 1629 \\
\hline Jatropha podagrica Hook. & $\mathrm{S}$ & 1630 \\
\hline Micrococca mercurialis Benth. & $\mathrm{H}$ & 1652 \\
\hline Pedilanthus tithymaloides (L.) Poit. & $\mathrm{S}$ & 1668 \\
\hline Ricinus communis $\mathrm{L}$. & S & 1690 \\
\hline Tragia involucrata $\mathrm{L}$. & $\mathrm{HC}$ & 1722 \\
\hline \multicolumn{3}{|l|}{ FABACEAE } \\
\hline Abrus precatorius L. & $\mathrm{L}$ & 1501 \\
\hline Acacia auriculiformis A.Cunn. ex Benth. & $\mathrm{T}$ & 1503 \\
\hline Adenanthera pavonina L. & $\mathrm{T}$ & 1507 \\
\hline Albizia lebbeck (L.) Benth. & $\mathrm{T}$ & 1512 \\
\hline Albizia saman (Jacq.) F.Muell. & $\mathrm{T}$ & 1513 \\
\hline Bauhinia racemosa Lam. & $\mathrm{T}$ & 1533 \\
\hline
\end{tabular}

TABLE 1. CONTINUED.

\begin{tabular}{|c|c|c|}
\hline TAXON & $\begin{array}{l}\text { LIFE- } \\
\text { FORM }\end{array}$ & $\begin{array}{c}\text { VOUCHER } \\
\text { NO. }\end{array}$ \\
\hline Butea monosperma (Lam.) Taub. In Engl. \& Prantl & $\mathrm{T}$ & 1541 \\
\hline Caesalpinia coriaria (Jacq.) Willd. & $\mathrm{T}$ & 1542 \\
\hline Caesalpinia pulcherrima (L.) Sw. & $\mathrm{S}$ & 1543 \\
\hline Cassia fistula $\mathrm{L}$. & $\mathrm{T}$ & 1554 \\
\hline Cassia hirsuta L. & $\mathrm{H}$ & 1745 \\
\hline Cassia occidentalis L. & $\mathrm{H}$ & 1746 \\
\hline Cassia roxburghii DC. & $\mathrm{T}$ & 1555 \\
\hline Cassia siamea Lam. & $\mathrm{T}$ & 1556 \\
\hline Clitoria ternatea $\mathrm{L}$. & $\mathrm{HC}$ & 1567 \\
\hline Crotalaria trifoliastrum Willd. & $\mathrm{H}$ & 1744 \\
\hline Delonix regia (Bojer) Raf. & $\mathrm{T}$ & 1586 \\
\hline Desmodium triflorum (L.) DC. & $\mathrm{H}$ & 1587 \\
\hline Erythrina variegata $\mathrm{L}$. & $\mathrm{T}$ & 1593 \\
\hline Gliricidia sepium (Jacq.) Kunth & $\mathrm{T}$ & 1604 \\
\hline Leucaena leucocephala (Lam.) de Wit. & $\mathrm{T}$ & 1640 \\
\hline Mimosa pudica L. & $\mathrm{H}$ & 1654 \\
\hline Peltophorum pterocarpum (DC.) Baker ex. K.Heyne & $\mathrm{T}$ & 1669 \\
\hline Pithecellobium dulce (Roxb.) Benth. & $\mathrm{T}$ & 1677 \\
\hline Pongamia pinnata Merr. & $\mathrm{T}$ & 1761 \\
\hline Prosopis juliflora (Sw.) Dc. & $\mathrm{T}$ & 1682 \\
\hline Rhynchosia cana DC. & $\mathrm{HC}$ & 1689 \\
\hline Senna alata (L.) Roxb. & $\mathrm{S}$ & 1697 \\
\hline Senna sophera (L.) Roxb. & $\mathrm{S}$ & 1698 \\
\hline Tamarindus indica L. & $\mathrm{T}$ & 1710 \\
\hline Tephrosia purpurea (L.) Pers. & $\mathrm{H}$ & 1713 \\
\hline \multicolumn{3}{|l|}{ GISEKIACEAE } \\
\hline Gisekia pharnaceoides L. & $\mathrm{H}$ & 1603 \\
\hline \multicolumn{3}{|l|}{ ICACINACEAE } \\
\hline Mappia foetida Miers & $\mathrm{S}$ & 1648 \\
\hline \multicolumn{3}{|l|}{ LAMIACEAE } \\
\hline Leucas aspera Link & $\mathrm{H}$ & 1641 \\
\hline Ocimum americanum L. & $\mathrm{H}$ & 1664 \\
\hline Ocimum tenuiflorum L. & $\mathrm{H}$ & 1665 \\
\hline \multicolumn{3}{|l|}{ LECYTHIDACEAE } \\
\hline Couroupita guianensis Aubl. & $\mathrm{T}$ & 1577 \\
\hline \multicolumn{3}{|l|}{ LYTHRACEAE } \\
\hline Lawsonia inermis L. & $\mathrm{T}$ & 1639 \\
\hline Punica granatum L. & $\mathrm{S}$ & 1685 \\
\hline \multicolumn{3}{|l|}{ MALVACEAE } \\
\hline Abutilon indicum (L.) Sweet & $\mathrm{S}$ & 1502 \\
\hline Bombax ceiba L. & $\mathrm{T}$ & 1538 \\
\hline Guazuma ulmifolia Lam. & $\mathrm{T}$ & 1612 \\
\hline Hibiscus rosa-sinensis $\mathrm{L}$. & $S$ & 1618 \\
\hline Hibiscus schizopetalus Hook.f. & $\mathrm{S}$ & 1619 \\
\hline Hibiscus tiliaceus $\mathrm{L}$. & $\mathrm{T}$ & 1620 \\
\hline Hibiscus vitifolius L. & $\mathrm{S}$ & 1621 \\
\hline Melochia corchorifolia L. & $\mathrm{S}$ & 1651 \\
\hline Pterospermum canescens Roxb. & $\mathrm{T}$ & 1684 \\
\hline Sida acuta Burm.f. & $\mathrm{H}$ & 1700 \\
\hline
\end{tabular}


TABLE 1. ContinUed.

\begin{tabular}{|c|c|c|}
\hline TAXON & $\begin{array}{l}\text { LIFE- } \\
\text { FORM }\end{array}$ & $\begin{array}{c}\text { VOUCHER } \\
\text { NO. }\end{array}$ \\
\hline Sida rhombifolia L. & $\mathrm{H}$ & 1742 \\
\hline Sterculia foetida $\mathrm{L}$. & $\mathrm{T}$ & 1706 \\
\hline Thespesia populnea (L.) Correa & $\mathrm{T}$ & 1716 \\
\hline Triumfetta rhomboidea Jacq. & $\mathrm{H}$ & 1727 \\
\hline Triumfetta rotundifolia Lam. & $\mathrm{H}$ & 1743 \\
\hline \multicolumn{3}{|l|}{ MARTYNIACEAE } \\
\hline Martynia annua $\mathrm{L}$. & $\mathrm{H}$ & 1650 \\
\hline \multicolumn{3}{|l|}{ Meliaceae } \\
\hline Azadirachta indica A.Juss. & $\mathrm{T}$ & 1525 \\
\hline \multicolumn{3}{|l|}{ MENISPERMACEAE } \\
\hline Tiliacora acuminata Miers & $\mathrm{L}$ & 1718 \\
\hline Tinospora cordifolia (Willd.) Hook.f. \& Thomson & $\mathrm{L}$ & 1719 \\
\hline \multicolumn{3}{|l|}{ MOLLUGinACEAE } \\
\hline Mollugo pentaphylla L. & $\mathrm{H}$ & 1747 \\
\hline \multicolumn{3}{|l|}{ MORACEAE } \\
\hline Artocarpus heterophyllus Lam. & $\mathrm{T}$ & 1523 \\
\hline Ficus benghalensis L. & $\mathrm{T}$ & 1596 \\
\hline Ficus elastica Roxb. & $\mathrm{T}$ & 1597 \\
\hline Ficus racemosa $\mathrm{L}$. & $\mathrm{T}$ & 1598 \\
\hline Ficus religiosa $\mathrm{L}$. & $\mathrm{T}$ & 1599 \\
\hline Ficus tomentosa Roxb. ex Willd. & $\mathrm{T}$ & 1600 \\
\hline Streblus asper Lour. & $\mathrm{T}$ & 1707 \\
\hline \multicolumn{3}{|l|}{ MORINGACEAE } \\
\hline Moringa pterygosperma Gaertn. & $\mathrm{T}$ & 1657 \\
\hline \multicolumn{3}{|l|}{ MUSACEAE } \\
\hline Musa paradisiaca L. & $\mathrm{S}$ & 1661 \\
\hline Ravenala madagascariensis J.F.Gmel. & $\mathrm{T}$ & 1688 \\
\hline \multicolumn{3}{|l|}{ MYRTACEAE } \\
\hline Callistemon citrinus (Curtis) Stapf & $\mathrm{T}$ & 1544 \\
\hline Psidium guajava L. & $\mathrm{T}$ & 1683 \\
\hline Syzygium cumini (L.) Skeels & $\mathrm{T}$ & 1708 \\
\hline \multicolumn{3}{|l|}{ NYCTAGINACEAE } \\
\hline Boerhavia diffusa L. & $\mathrm{H}$ & 1536 \\
\hline Boerhavia erecta L. & $\mathrm{H}$ & 1537 \\
\hline Bougainvillea spectabilis Willd. & $\mathrm{L}$ & 1540 \\
\hline Pisonia alba Span. & $\mathrm{T}$ & 1676 \\
\hline \multicolumn{3}{|l|}{ OCHNACEAE } \\
\hline Gomphia serrata (Gaertn.) Kanis & $\mathrm{S}$ & 1607 \\
\hline \multicolumn{3}{|l|}{ OLEACEAE } \\
\hline Jasminum sambac (Soland.) & $\mathrm{L}$ & 1626 \\
\hline Nyctanthes arbor-tristis L. & $\mathrm{T}$ & 1663 \\
\hline \multicolumn{3}{|l|}{ PASSIFLORACEAE } \\
\hline Passiflora foetida $\mathrm{L}$. & $\mathrm{HC}$ & 1666 \\
\hline \multicolumn{3}{|l|}{ Phyllanthaceae } \\
\hline Phyllanthus acidus (L.) Skeels & $\mathrm{T}$ & 1672 \\
\hline Phyllanthus emblica L. & $\mathrm{T}$ & 1673 \\
\hline Phyllanthus maderaspatensis L. & $\mathrm{H}$ & 1674 \\
\hline Phyllanthus reticulatus Poir. & $\mathrm{S}$ & 1675 \\
\hline
\end{tabular}

TABle 1. Continued.

\begin{tabular}{|c|c|c|}
\hline TAXON & $\begin{array}{l}\text { LIFE- } \\
\text { FORM }\end{array}$ & $\begin{array}{c}\text { VOUCHER } \\
\text { NO. }\end{array}$ \\
\hline Rivina humilis L. & $\mathrm{H}$ & 1691 \\
\hline \multicolumn{3}{|l|}{ Plumbaginaceae } \\
\hline Plumbago capensis Thunb. & $\mathrm{H}$ & 1678 \\
\hline Plumbago zeylanica L. & $\mathrm{H}$ & 1679 \\
\hline \multicolumn{3}{|l|}{ POACEAE } \\
\hline Bambusa balcooa Roxb. & $\mathrm{S}$ & 1527 \\
\hline Bambusa bambos (L.) Voss & $\mathrm{S}$ & 1528 \\
\hline Bambusa nutans Wall. ex Munro & $\mathrm{S}$ & 1529 \\
\hline Bambusa vulgaris Schrad. ex J.C. Wendl. var. striata & $\mathrm{S}$ & 1530 \\
\hline Bambusa vulgaris Schrad. ex J.C. Wendl. var. wamin & $\mathrm{S}$ & 1531 \\
\hline Chloris barbata $\mathrm{Sw}$. & $\mathrm{H}$ & 1561 \\
\hline Cynodon dactylon (L.) Pers. & $\mathrm{H}$ & 1581 \\
\hline Echinochloa colona (L.) Link. & $\mathrm{H}$ & 1758 \\
\hline Oplismenus compositus (L.) P.Beauv. & $\mathrm{H}$ & 1759 \\
\hline Setaria verticillata (L.) P.Beauv. & $\mathrm{H}$ & 1699 \\
\hline Sporobolus coromandelianus (Retz.) Kunth & $\mathrm{H}$ & 1705 \\
\hline \multicolumn{3}{|l|}{ Polygonaceae } \\
\hline Antigonon leptopus Hook. \& Arn. & $\mathrm{L}$ & 1520 \\
\hline Coccoloba uvifera L. & $\mathrm{T}$ & 1569 \\
\hline \multicolumn{3}{|l|}{ Proteaceae } \\
\hline Grevillea robusta A.Cunn. ex R.Br. & $\mathrm{T}$ & 1610 \\
\hline \multicolumn{3}{|l|}{ Putranjivaceae } \\
\hline Drypetes roxburghii (Wall.) Hurus. & $\mathrm{T}$ & 1590 \\
\hline \multicolumn{3}{|l|}{ RHAMNACEAE } \\
\hline Ventilago maderaspatana Gaertn. & $\mathrm{L}$ & 1730 \\
\hline Ziziphus mauritiana Lam. & $\mathrm{T}$ & 1734 \\
\hline \multicolumn{3}{|l|}{ RUBIACEAE } \\
\hline Canthium coromandelicum (Burm.f.) Alston & $\mathrm{S}$ & 1547 \\
\hline Hamelia patens Jacq. & $\mathrm{S}$ & 1614 \\
\hline Hedyotis umbellata Lam. & $\mathrm{H}$ & 1615 \\
\hline Ixora coccinea $\mathrm{L}$. & $\mathrm{S}$ & 1624 \\
\hline Ixora pavetta Andrews & $\mathrm{T}$ & 1625 \\
\hline Morinda coreia Buch.-Ham. & $\mathrm{T}$ & 1656 \\
\hline Pavetta indica L. & $\mathrm{S}$ & 1667 \\
\hline Spermacoce ocymoides Burm.f. & $\mathrm{H}$ & 1704 \\
\hline \multicolumn{3}{|l|}{ RUTACEAE } \\
\hline Aegle marmelos Correa & $\mathrm{T}$ & 1509 \\
\hline Citrus aurantiifolia (Christm.) Swingle & $\mathrm{T}$ & 1563 \\
\hline Citrus medica L. & $\mathrm{T}$ & 1564 \\
\hline Limonia acidissima L. & $\mathrm{T}$ & 1642 \\
\hline Murraya koenigii Spreng. & $\mathrm{T}$ & 1659 \\
\hline Murraya paniculata (L.) Jack & $\mathrm{T}$ & 1660 \\
\hline Toddalia asiatica Lam. & $\mathrm{S}$ & 1720 \\
\hline \multicolumn{3}{|l|}{ SAlicaceae } \\
\hline Flacourtia indica (Burm.f.) Merr. & $\mathrm{S}$ & 1602 \\
\hline \multicolumn{3}{|l|}{ SALVADORACEAE } \\
\hline Azima tetracantha Lam. & $\mathrm{S}$ & 1526 \\
\hline \multicolumn{3}{|l|}{ SAPINDACEAE } \\
\hline Allophylus cobbe (L.) Raeusch. & S & 1514 \\
\hline
\end{tabular}


TABLE 1. ContinUed.

\begin{tabular}{|c|c|c|}
\hline TAXON & $\begin{array}{l}\text { LIFE- } \\
\text { FORM }\end{array}$ & $\begin{array}{c}\text { VOUCHER } \\
\text { NO. }\end{array}$ \\
\hline Cardiospermum halicacabum L. & $\mathrm{L}$ & 1550 \\
\hline Dodonaea viscosa Jacq. & S & 1589 \\
\hline Filicium decipiens Thwaites & $\mathrm{T}$ & 1601 \\
\hline Sapindus emarginatus Vahl. & $\mathrm{T}$ & 1695 \\
\hline \multicolumn{3}{|l|}{ SAPOTACEAE } \\
\hline Madhuca indica J.F.Gmel. & $\mathrm{T}$ & 1644 \\
\hline Manilkara hexandra Dubard & $\mathrm{T}$ & 1646 \\
\hline Manilkara zapota (L.) P.Royen & $\mathrm{T}$ & 1647 \\
\hline Mimusops elengi $\mathrm{L}$. & $\mathrm{T}$ & 1655 \\
\hline \multicolumn{3}{|l|}{ SCROPHULARIACEAE } \\
\hline Scoparia dulcis L. & $\mathrm{H}$ & 1754 \\
\hline \multicolumn{3}{|l|}{ SiMAROUBACEAE } \\
\hline Simarouba glauca DC. & $\mathrm{T}$ & 1701 \\
\hline \multicolumn{3}{|l|}{ SOLANACEAE } \\
\hline Datura innoxia Mill. & $\mathrm{H}$ & 1584 \\
\hline Solanum americanum Mill. & $\mathrm{H}$ & 1702 \\
\hline Solanum trilobatum $\mathrm{L}$. & $\mathrm{L}$ & 1753 \\
\hline \multicolumn{3}{|l|}{ TURNERACEAE } \\
\hline Turnera ulmifolia $\mathrm{L}$. & $\mathrm{H}$ & 1728 \\
\hline \multicolumn{3}{|l|}{ VERBENACEAE } \\
\hline Clerodendrum inerme (L.) Gaertn. & $\mathrm{S}$ & 1566 \\
\hline Gmelina arborea Roxb. & $\mathrm{T}$ & 1606 \\
\hline Lantana camara L. & $\mathrm{S}$ & 1638 \\
\hline Premna latifolia Roxb. & $\mathrm{T}$ & 1681 \\
\hline Tectona grandis L.f. & $\mathrm{T}$ & 1712 \\
\hline Vitex negundo L. & $\mathrm{T}$ & 1731 \\
\hline Waltheria indica L. & $\mathrm{H}$ & 1732 \\
\hline \multicolumn{3}{|l|}{ VIOLACEAE } \\
\hline Hybanthus enneaspermus (L.) F.Muell. & $\mathrm{H}$ & 1622 \\
\hline \multicolumn{3}{|l|}{ VITACEAE } \\
\hline Cissus quadrangularis $\mathrm{L}$. & $\mathrm{L}$ & 1562 \\
\hline \multicolumn{3}{|l|}{ ZYGOPHYLLACEAE } \\
\hline Guaiacum officinale L. & $\mathrm{T}$ & 1611 \\
\hline Tribulus lanuginosus L. & $\mathrm{H}$ & 1724 \\
\hline
\end{tabular}

Acknowledgments: The first author is thankful to Department of Science and Technology, Government of India, New Delhi for their financial support through INSPIRE Fellowship (Ref. no: DST/INSPIRE Fellowship/2009). The authors are thankful to Prof. M.P. Murugesan and Prof. Dr. K. Ajithadoss for their constant encouragement and support during the course of this study. The authors record their gratitude for the Head of the Department of Botany and the Principal, Pachaiyappa's College for granting permission to undertake this study.

\section{Literature Cited}

Beckett, K.P., P. Freer-Smith and G. Taylor. 2000. Effective tree species for local air-quality management. Journal of Arboriculture 26: 12-19.

Blasco, F. and P. Legris. 1973. Dry evergreen forests of Point Calimere and Marakanam. Journal of Bombay Natural History Society 70: 279-294.

Bunyavejchewin, S. 1999. Structure and dynamics in seasonal dry evergreen forest in northeastern Thailand. Journal of Vegetation Science 10: 787-792.

Champion, H.G. and S.K. Seth. 1968. Revised survey of the forest types of India. New Delhi: Manager of Publications.

Chennai District. 2009. Electronic database accessible at http://www. chennai.tn.nic.in. Captured on 14 July 2009.
Chow, W.T.L. and M. Roth. 2006. Temporal dynamics of the urban heat island of Singapore. International Journal of Climatology 26: 22432260 .

Cy, J. 2006. Formulaic expert method to integrate evaluation and valuation of heritage trees in compact city. Environmental Monitoring and Assessment 116: 53-80.

Fernandez-Juricic, E. 2000. Avifaunal use of wooded streets in an urban landscape. Conservation Biology 14: 513-521.

Fyson, P.F. 1921. Madras Flowers. The botanical bulletin of the Presidency College, Madras. 1: 90-100.

Gamble, J.S. and C.E.C. Fischer. 1921-35. Flora of the Presidency of Madras. 3 Vols. London: Adlard and Son Ltd. 2017 p.

Giles-Lal, D. and C. Livingstone. 1978. Campus flora of Madras Christian College. Madras: The Balussery Press. 78 p.

Henry, A.N., V. Chitra and N.P. Balakrishnan. 1989. Flora of Tamil Nadu, India. Series I: Analysis. Vol. 3. Coimbatore: Botanical Survey of India. $171 \mathrm{p}$.

Henry, A.N., G.R. Kumari and V. Chitra. 1987. Flora of Tamil Nadu, India. Series I: Analysis. Vol. 2. Coimbatore: Botanical Survey of India. 258 p.

IPNI 2009. International Plant Names Index. Electronic database accessible at http://www.ipni.org/ipni/plantnamesearchpage.do. Captured on 16 July 2009.

IUCN 2009. IUCN Red List of Threatened Species. Version 2009.1. Electronic database accessible at http://www.iucnredlist.org. Captured on 17 July 2009.

Kelly, D.L., E.V.J. Tanner, V. Kapos. T.A. Dickinson, G.A. Goodfriend and P. Fairbairn. 1988. Jamaican limestone forests: floristics, structure and environment of three examples along a rainfall gradient. Journal of Tropical Ecology 4: 121-156.

Mani, M.S. 1974. (ed.) Ecology and biogeography in India. The Hague: W. Junk. 773 p.

Mani, S. and N. Parthasarathy. 2005. Biodiversity assessment of trees in five inland tropical dry evergreen forests of peninsular India. Systematics and Biodiversity 3: 1-12.

Matthew, K.M. 1991. An excursion Flora of Central Tamil Nadu. Thiruchirappalli: Rapinat Herbarium. 682 p.

Mayuranathan, P.V. 1929. The flowering plants of Madras city and its immediate neighbourhood. Bulletin of the Madras Government Museum 2: $1-345$.

Mayuranathan, P.V. 1994. The flowering plants of Madras city and its immediate neighbourhood. Bulletin of the Madras Government Museum 10: 1-400.

Mc Pherson, E.G., D. Nowak, G. Heisler, S. Grimmond, C. Souch, R. Grant and R. Rowntree. 1997. Quantifying urban forest structure, function and value: the Chicago Urban Forest Climate Project. Urban Ecosystems 49-61.

Meher-Homji, V.M. 1974. On the origin of tropical dry evergreen forest of south India. International Journal of Ecology and Environmental Science 1: 19-39.

Miller, R.W. 1996. Urban Forestry: Planning and Managing Urban Green spaces. Englewood Cliffs: Prentice-Hall. 502 p.

Nair, N.C. and A.N. Henry. 1987. Flora of Tamil Nadu, India. Series I: Analysis. Vol. 1. Coimbatore: Botanical Survey of India. 184 p.

Parthasarathy, N., M. Arthur Selwyn and M. Udayakumar. 2008. Tropical dry evergreen forests of peninsular India: ecology and conservation significance. Tropical Conservation Science 1: 89-110.

Parthasarathy, N and R. Karthikeyan. 1997. Plant biodiversity inventory and conservation of two tropical dry evergreen forests on the Coromandel coast, south India. Biodiversity and Conservation. 6: 10631083.

Rao, T.A. and V.M. Meher-Homji. 1993. Dry coastal ecosystem of Indian subcontinent and islands; p. 151-164 In E. Van der Maarel (ed.) Ecosystems of the World: Dry Coastal Ecosystems - Africa, America, Asia and Oceania. Amsterdam: Elsevier.

Reddy, M.S. and N. Parthasarathy. 2003. Liana diversity and distribution in four tropical dry evergreen forests on the Coromandel coast of south India. Biodiversity and Conservation 12: 1609 -1627.

Sebastine, K.M. and J.L. Ellis. 1967. A contribution to the vascular flora of Vedharanyam and Talaignayar Reserve forests, Tanjore district, Madras state. Bulletin of Botanical Survey of India 9: 190-200.

Sodhi N.S., M.R.C. Posa, T.M. Lee, D. Bickford, L.P. Koh and B.W. Brook. 2010. The state and conservation of Southeast Asian biodiversity. Biodiversity and Conservation 19(2): 317-328.

Tamil Nadu Government. 2009. Electronic database accessible at http:// www.chennai.tn.nic.in/chndistprof.htm\#geog. Captured on 12 July 2009.

Thaiutsa, B., L. Puangchit, R. Kjelgren and W. Arunpraparut. 2008. Urban green space, street tree and heritage large tree assessment in Bangkok, Thailand. Urban Forestry and Urban Greening 7: 219-229.

Udayakumar, M. and N. Parthasarathy. 2010. Angiosperms, tropical dry evergreen forests of southern Coromandel coast, India. Check List 6: 368-381. 
Venkateswaran, R. and N. Parthasarathy. 2003. Tropical dry evergreen forests on the Coromandel Coast of India: Structure, composition and human disturbance. Ecotropica 9: 45-58.

Venkateswaran, R. and N. Parthasarathy. 2005. Tree population changes in a tropical dry evergreen forest of south India over a decade (19922002). Biodiversity and Conservation 14: 1335-1344.
RECEIVED: August 2009

LAST REVISED: September 2010

ACCEPTED: September 2010

PUBLISHED ONLINE: January 2011

EDITORIAL RESPONSIBILITY: Frederico Augusto Guimarães Guilherme 